\title{
Regenerated hydrogel electrolyte towards an all-gel supercapacitor
}

\author{
Xian He, Dongbei Wu*, Yinghui Shang, Hongdou Shen, Songyan Xi, Xia Wang, Wenjun Li and \\ Qigang Wang*
}

\begin{abstract}
Electrolyte regeneration is an important goal for environmental protection and sustainable development efforts. Herein, we report a facile strategy inspired by the transformation of edible dough from flour to regenerate hydrogel electrolytes from their dehydrated copolymer granules (CGs) via direct addition of water or salt solution. With the aid of heating, this procedure is efficient, relatively quick, and easily implemented. The dehydrated CGs are lightweight, reusable and stable under long-term storage. Even after $5 \mathrm{cy}$ cles of dehydration and regeneration, the regeneration efficiency of the hydrogel electrolytes, as evaluated based on retention of mechanical strength, is over $60 \%$. The regenerated electrolytes possess considerable ionic conductivity, reprocessability, and 3D-printability. Furthermore, an all-gel supercapacitor assembled from the regenerated hydrogel electrolyte and activated carbon electrode with CGs as binder demonstrates excellent interfacial compatibility. The assembled all-gel supercapacitor can maintain $98.7 \%$ of its original specific capacitance after 100 bending tests, and can operate in a wide temperature range spanning from -15 to $60^{\circ} \mathrm{C}$. This work may provide a new access to the development of renewable materials for various applications in the fields of intelligent devices, wearable electronics and soft robotics.
\end{abstract}

Keywords: hydrogel electrolyte, renewable, copolymer granules, supercapacitor

\section{INTRODUCTION}

Increasing demands on energy storage devices for routine applications has motivated substantial interest in the research and development of flexible and wearable electronics, including supercapacitors and solid-state batteries [1-3]. As a key component of flexible energy devices, gel electrolytes have received exceptional attention due to their dual roles of electrolyte and separator $[2,4]$. Because desirable energy storage devices demand high power density and long operating lifetime, the gel electrolytes ideally possess a high ionic migration rate, excellent interfacial properties, and reasonable mechanical strength [5-7]. Moreover, the inevitable mechanical stresses and deformations suffered by most energy devices can ultimately lead to permanent damage of device appearance and microstructure. Therefore, a highly desirable property of the gel electrolytes is selfhealing ability which enables automatic restoration of original performance after mechanical damage [8-10]. Regeneration ability is also valuable from a sustainability perspective, as a better choice to recycle waste gel electrolytes mitigates waste and resource consumption.

Gel electrolytes are generally composed of polymeric materials and electrolyte salt, with the former acting as a matrix and the latter providing mobile ions. Compared with conventional gel electrolytes comprised of polyvinyl alcohol (PVA) hydrogels $[11,12]$, more recently developed hydrogels can be endowed with desirable physical and chemical properties. For example, polyacrylamide (PAM)-based hydrogels exhibit good flexibility and water retention [13,14], while zwitterion hydrogels feature promising zwitterion conductivity [15-17], all of which offer an ideal platform for constructing flexible and self-healing electronics. Meanwhile, the expanding development of gel electrolytes from traditional aqueous gel electrolytes to non-aqueous gel electrolytes has served to facilitate significantly enhanced ion migration rates and mechanical properties of electrolytes, thereby greatly improving their electrochemical performance [18-20]. However, the synthesis routes for functional PAMbased hydrogels or zwitterion gel electrolytes feature complicated design and operation elements. Accordingly, tedious polymerization procedures and stringent experimental conditions severely limit the volume production [21,22]. Moreover, little consideration has been paid to the issue of the electrolyte regeneration. Therefore, although great progress has been made towards the development of efficient gel electrolytes, persistent efforts are still needed to identify a convenient and quick procedure to restore gel electrolyte properties.

Edible dough, comprised of flour mixed with salts and water, is a household food whose shape and functionality can be easily reassembled and reconfigured [23-25]. However, to date, few efforts have focused on the regeneration of synthetic materials from tiny building blocks via direct addition of water. Among these few relevant studies, Gong et al. $[21,26]$ were the first to employ [3-(methacryloylamino)propyl]-trimethylammonium chloride (MPTC) and sodium $p$-styenesulfonate (NaSS) to construct a tough self-glued polyion complexes (PIC) hydrogel prepared from its microgel granules. Polyion interactions between positively charged MPTC and negatively charged NaSS are considered to be the driving force for rebuilding the hydrogels. Recently, $\mathrm{Qu}$ et al. [27] reported a tough double network polyacrylamide-sodium alginate hydrogel from selfregenerative powders, in which hydrogen bonds play a crucial role in regeneration. Most importantly, the regeneration of these hydrogels is quite efficient, easily operated and time-saving, comprising a feasible strategy which can be sustainably applied to develop synthetic functional materials from their granules. 
Inspired by these pioneering studies, herein we reported the leveraging of multiple interactions including hydrophilic hydrogen bonds and polyion interactions as well as hydrophobic polymer chain entanglement to construct a tough hydrogel via the random copolymerization of [3-(methacryloylamino)-propyl] trimethylammonium chloride (MAPTAC) and methyl acrylic acid (MAA). Dehydration and grinding of the hydrogel yielded white copolymer granules (CGs) which were employed as starting materials to regenerate the gel electrolytes for subsequent experiments. We found that these CGs are lightweight, stable, and can be easily stored for a long time. Notably, these CGs can efficiently regenerate gel electrolytes via simply mixing salt and water with them, and can maintain good mechanical properties even after 5 rounds of recycling. Dynamic interactions including the hydrogen bonds and the polyion interactions between $-\mathrm{N}\left(\mathrm{CH}_{3}\right)_{3}{ }^{+}$cations and $-\mathrm{COO}^{-}$anions play an important role in the electrolyte regeneration, mechanical robustness, and autonomous healing. Meanwhile, the introduction of salt ions proportionally into the CGs ensures high ionic conductivity and stable electrochemical compliance of the regenerated hydrogel electrolyte (RHE). Importantly, these CGs not only self-glue to each other, but also act as a binder adhering the charged active carbon (AC) powders to form a flexible AC/CGs composite electrode. Adopting a sandwiched structure for the supercapacitor, the RHE can be tightly bonded between the composite electrodes and integrated into a dynamic polymer network which significantly mitigates the interfacial resistance between the electrolyte and electrode, even as the mechanical and electrochemical stabilities are maintained across 100 bending iterations. It is anticipated that this work may provide new insight into dynamic interactions and promote the development of renewable materials for a wide range of applications, such as smart wearable electronics, flexible energy devices, and artificial skin.

\section{EXPERIMENTAL SECTION}

\section{Materials}

MAPTAC and polytetrafluoroethylene (60 wt\%) were purchased from Aladdin Bio-tech Co., Ltd. MAA and a-ketoglutaric acid were purchased from J\&K Scientific Ltd. Sodium sulfate $\left(\mathrm{Na}_{2} \mathrm{SO}_{4}\right)$, hydrochloric acid $(\mathrm{HCl})$, sodium hydroxide $(\mathrm{NaOH})$ and deuteroxide $\left(\mathrm{D}_{2} \mathrm{O}\right)$ were purchased from Sinopharm Chemical Reagent Co., Ltd. AC powder (YP80F, $2100 \mathrm{~m}^{2} \mathrm{~g}^{-1}$ ) was purchased from Kuraray Co. Acetylene black was purchased from Shanghai 3F New Material Co., Ltd. All reagents were used without further purification.

\section{Preparation of the original hydrogel electrolyte (OHE) and the CGs}

In a typical preparation procedure, $1.5 \mathrm{~g}$ MAPTAC, $0.4 \mathrm{~g}$ MAA and $0.03 \mathrm{~g}$ a-ketoglutaric acid were added under stirring to $1.1 \mathrm{~g}$ distilled water or $\mathrm{Na}_{2} \mathrm{SO}_{4}$ solution to form a precursor solution. Next, the precursor solution was exposed to UV irradiation for about $100 \mathrm{~min}$ at room temperature, during which time the solution was under continuous $\mathrm{pH}$ monitoring. The obtained product was designated the OHE.

The CGs were prepared from the as-prepared OHE. The OHE was frozen in a refrigerator for $12 \mathrm{~h}$ and subsequently dehydrated in a lyophilizer for an additional $72 \mathrm{~h}$ to obtain white aerogels. The aerogels were then ground by a blender for $5 \mathrm{~min}$ and filtered through a 300-mesh sieve.

\section{Fabrication of the RHE}

Regeneration of the hydrogel electrolyte was accomplished by directly adding $1.2 \mathrm{~g}$ of the as-prepared CGs to $1.8 \mathrm{~g}$ distilled water or $\mathrm{Na}_{2} \mathrm{SO}_{4}$ solution of various $\mathrm{pH}$ conditions and stirring the resulting dispersion at $60^{\circ} \mathrm{C}$ for $10 \mathrm{~min}$. Recycling of the RHE required only the addition of water to the CGs containing $\mathrm{Na}_{2} \mathrm{SO}_{4}$.

\section{Assembly of the all-gel supercapacitor}

Gel electrodes were obtained from the mixture of the CGs, AC powder, and acetylene black (mass ratio 1:3:0.8) in $0.25 \mathrm{~mol} \mathrm{~L}^{-1}$ $\mathrm{Na}_{2} \mathrm{SO}_{4}$ solution under gentle stirring for $3 \mathrm{~h}$.

The supercapacitors were fabricated using a typical sandwich structure, wherein the middle was comprised of the RHE with $1 \mathrm{~mol} \mathrm{~L}^{-1} \mathrm{Na}_{2} \mathrm{SO}_{4}$ and the sides were comprised of two composite gel electrodes. The assembled supercapacitors were subsequently heated for $10 \mathrm{~min}$ at $60^{\circ} \mathrm{C}$.

More details about the materials, the CG preparation, the RHE fabrication, the supercapacitor assembly, the material characterization and the electrochemical measurements of the electronic devices can be obtained from the Supplementary information.

\section{RESULTS AND DISCUSSION}

Fig. 1a shows the fabrication scheme for the RHE. Firstly, the OHE was prepared from random copolymerization of MAPTAC and MAA in a specified salt solution (Fig. 1a-i). Salt ion concentration in the solution ranged from 0 to $1 \mathrm{~mol} \mathrm{~L}^{-1}$, and no additional crosslinkers were used. Next, the OHE was freezedried to produce a white aerogel with a porous network structure (Fig. 1a-ii and Fig. S1). Grinding the aerogel into small particles obtained white CGs measuring tens of micrometres in diameter, as shown in Fig. 1a-iii and Fig. S2. We found the CGs to be lightweight and stable, even under long-term storage, during which the mass of CGs remained stable and the gel could be regenerated successfully (Fig. S3). The remarkable renewability of the gels was readily observed upon reconstruction into an RHE via addition of water or salt ions (Fig. 1a-iv, 1a-v). The regeneration time at $60^{\circ} \mathrm{C}$ was less than $10 \mathrm{~min}$. Accordingly, the CGs are defined as intermediate materials for the RHE formation. It is worth noting that there are no obvious differences with respect to respective appearance or microstructure of the $\mathrm{OHE}$ and RHE (Fig. S4). The formation process of the RHE from the CGs is quite similar to that of the edible dough from flour and water, and is very easily performed, even by children. The OHECGs-RHE cycle is completely reversible and can be repeated several times without extreme loss of mechanical or electrochemical performance, which may be attributed to the multifunctional physically crosslinked network (Fig. 1a-vi). This network structure endows the RHE with additional traits, such as adhesivity, reprocessability, and printability. Fig. 1a-vii and 1a-viii demonstrate that after heating, the RHE can be reversibly molded into a specific shape, such as petal-like and butterfly-like shapes.

Fig. 1b-d vividly depict the flexibility, adhesivity and printability of the RHE. The RHE can be modestly knotted, stretched, twisted and spread without suffering any obvious damage in appearance (Fig. 1b). They can be self-glued or adhered to the surface of other objects, such as metal and plastic products 


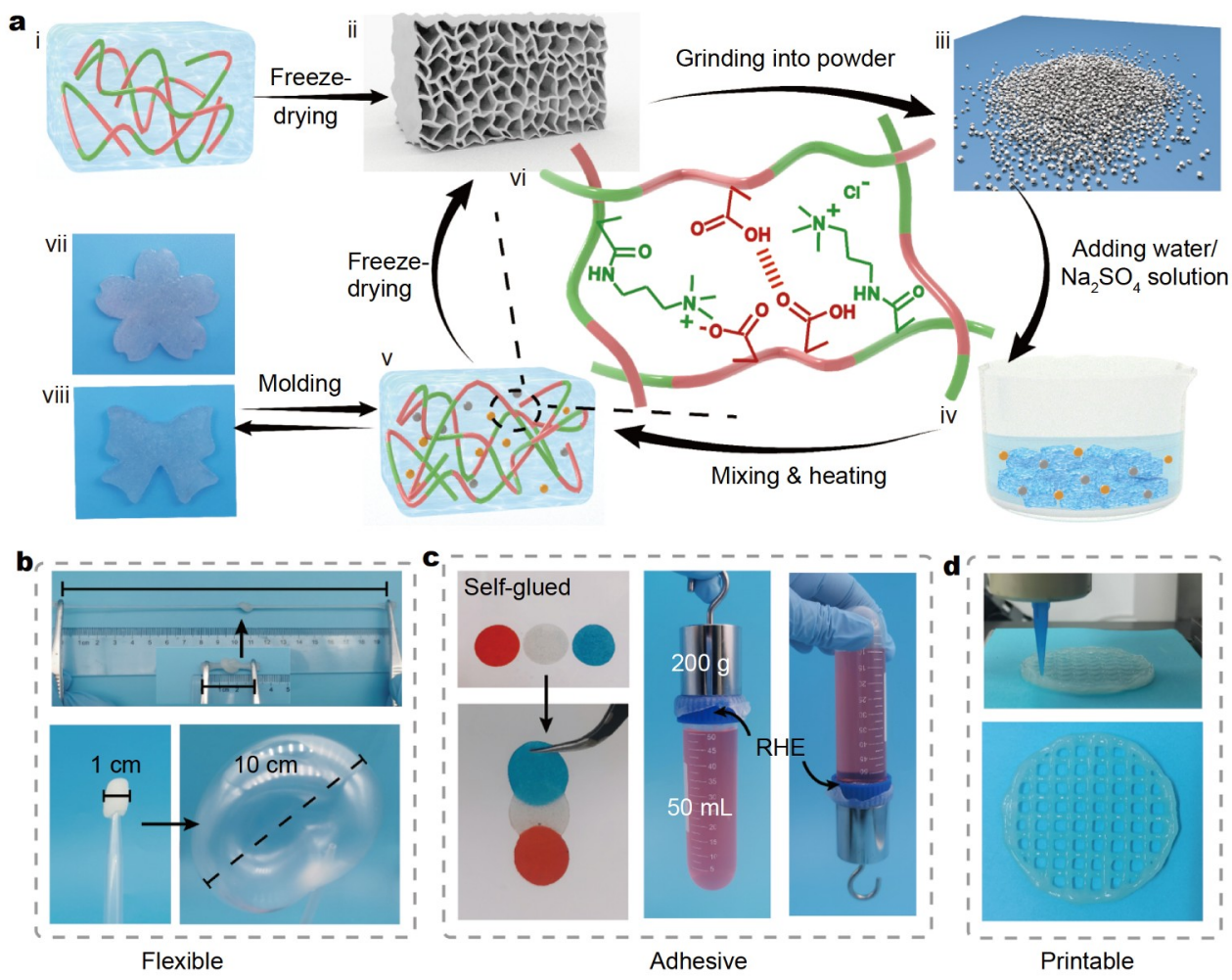

Figure 1 Preparation and demonstration of the RHE. (a) Fabrication scheme for the RHE. (i) The OHE. (ii) Aerogels obtained by freeze-drying. (iii) The CGs. (iv) A mixture of the CGs and $\mathrm{Na}_{2} \mathrm{SO}_{4}$ solution. (v) The RHE. (vi) The multifunctional physically crosslinked network structure of the RHE. (vii, viii) Thermo-mold of the RHE. (b) Flexibility of the RHE. (c) Adhesion of the RHE. (d) 3D-printability of the RHE.

(Fig. 1c). Fig. S5a shows that the storage moduli $\left(G^{\prime}\right)$ exceeds the loss moduli $\left(G^{\prime \prime}\right)$ over 0.1 to $30 \mathrm{~Hz}$ frequency range, and are unaffected by frequency, which indicated good stability of the RHE. Fig. S5b indicates that the moduli remain invariant in the temperature range of $25-50^{\circ} \mathrm{C}$, but gradually attenuate above $50^{\circ} \mathrm{C}$. Near $70^{\circ} \mathrm{C}$, the $G^{\prime}$ value equals to that of $G^{\prime \prime}$, suggesting that the RHE is in a flowing state and can be extruded as a kind of printing ink. Fig. 1d further confirms that the RHE can be made into various patterns by a $3 \mathrm{D}$ printer operated at $70^{\circ} \mathrm{C}$. Taken together, the above findings suggest that the hydrogel electrolyte regeneration via the CG reconstruction is facile, rapid and capable of fabricating flexible energy devices at an unprecedented level of convenience.

The quantitative characterizations of the RHE mechanical strength are available in Fig. 2. Fig. 2a shows that as $\mathrm{pH}$ decreases from 4.0 to 1.0 , the extension strain sharply increases from $127 \%$ to $2822 \%$, and fracture energy jumps from 15.4 to $322.4 \mathrm{~kJ} \mathrm{~m}^{-3}$ (Table $\mathrm{S} 1$ ). These relationships reveal that low $\mathrm{pH}$ can endow the RHE with outstanding stretchability and toughness, likely due to hydrogen bond contributions [28]. As Zeta potential data show the isoelectric point of the p(MAA-MAPTAC) copolymer particles is between 4.0 and 8.0 (Fig. S6), we can deduce that at $\mathrm{pH}$ below 4.0 , the $-\mathrm{N}\left(\mathrm{CH}_{3}\right)_{3}{ }^{+}$groups are protonated and ionization of $-\mathrm{COOH}$ groups is inhibited. High acidity can provide more binding sites for hydrogen bond formation, which thereby enhances the stretchability and toughness of the RHE. However, highly acidic conditions also promote significant repulsive forces from the protonated $-\mathrm{N}\left(\mathrm{CH}_{3}\right)_{3}{ }^{+}$ functional groups. These repulsions cannot be neglected, and may even displace some electrostatic interactions between positive and negative charges. As a result, the RHE stiffness under low $\mathrm{pH}$ conditions may be lower than that under high $\mathrm{pH}$ conditions. As shown in Fig. $2 \mathrm{~b}$, the $\mathrm{pH}$ decrease from 4.0 to 2.0 causes the compressive stress of the $\mathrm{RHE}$ at $90 \%$ strain to increase from 334 to $516 \mathrm{kPa}$. However, when the $\mathrm{pH}$ turns lower than 2.0, the compressive stress decreases from 516 to $210 \mathrm{kPa}$, indicating that low $\mathrm{pH}$ indeed promotes weakening of the RHE. Therefore, all subsequent experiments employed the RHE obtained at a $\mathrm{pH}$ of 2.0 to ensure the suitable mechanical performance. To evaluate the RHE regeneration ability, the regeneration efficiency was arbitrarily defined as the ratio of the RHE compressive modulus to those of the OHE. Our findings, presented in Fig. 2c, suggest that the compressive modulus values of the RHE are slightly lower than those of the OHE in the selected $\mathrm{pH}$ range. With increasing $\mathrm{pH}$, the regeneration efficiency of the RHE monotonously decreases. Approximately $90 \%$ recovery can be achieved at $\mathrm{pH} 2.0$, indicating that effective regeneration of the RHE.

The mechanical strength of the RHE is also significantly affected by addition of $\mathrm{Na}_{2} \mathrm{SO}_{4}$. Fig. $2 \mathrm{~d}$ depicts that as the salt concentration increases from 0 to $1 \mathrm{~mol} \mathrm{~L}^{-1}$, the tensile strain dramatically decreases from $1008 \%$ to $110 \%$, and the corresponding fracture modulus increases from 44.0 to $103.5 \mathrm{kPa}$. Taken together, these results suggest that low salinity is beneficial for the stretchability while high salinity improves the tensile strength of the RHE (Table S2). Fig. 2e shows that as the salt concentration increases from 0 to $1 \mathrm{~mol} \mathrm{~L}^{-1}$, the $\mathrm{RHE}$ compressive stress at $90 \%$ strain monotonically increases from 0.5 to $2.25 \mathrm{MPa}$, indicating that high salinity increases the RHE stiffness. Fig. $2 \mathrm{f}$ indicates that as the salt concentration increases 

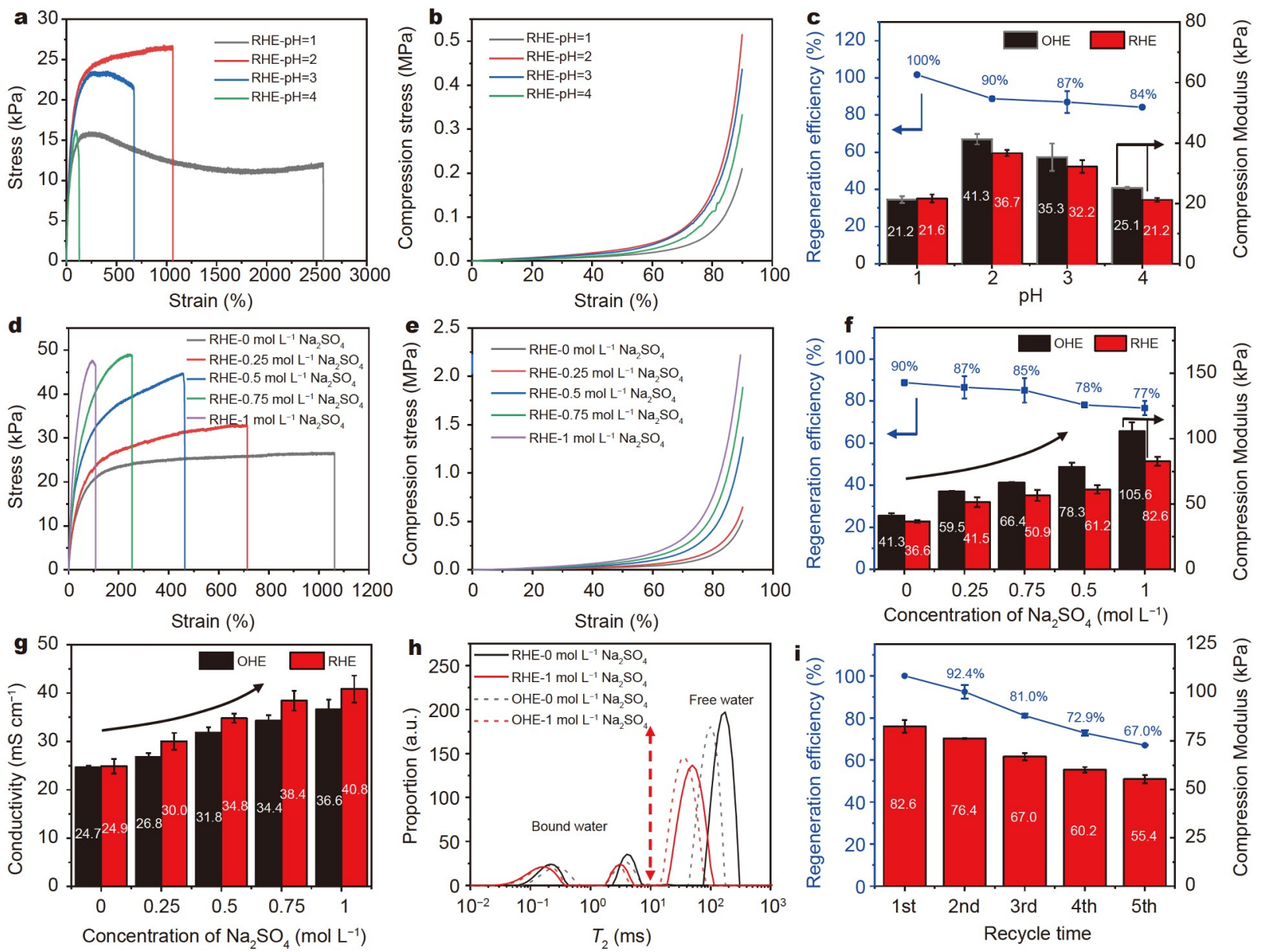

Figure 2 Mechanical characterizations of the OHE and RHE. (a) Tensile curves of the RHE with various pH values. (b) Compressive curves of the RHE at various $\mathrm{pH}$ values. (c) Compression moduli of both the OHE and RHE and regeneration efficiency at various pH values. (d) Tensile curves of the RHE for various $\mathrm{Na}_{2} \mathrm{SO}_{4}$ concentrations. (e) Compressive curves of the RHE for various $\mathrm{Na}_{2} \mathrm{SO}_{4}$ concentrations. (f) Compression moduli of both the OHE and $\mathrm{RHE}$ and regeneration efficiency for various $\mathrm{Na}_{2} \mathrm{SO}_{4}$ concentrations. (g) Ionic conductivities of the OHE and RHE for various $\mathrm{Na}_{2} \mathrm{SO}_{4}$ concentrations. (h) Low-field NMR inversion curves of water signals in various samples. (i) Regeneration efficiency and ionic conductivity of the RHE across 5 rounds of recycling.

from 0 to $1 \mathrm{~mol} \mathrm{~L} \mathrm{~L}^{-1}$, the relative compressive modulus of the OHE increases from 41.3 to $105.6 \mathrm{kPa}$, while that of the RHE increases from 36.4 to $82.6 \mathrm{kPa}$. However, across the same range, the regeneration efficiency slightly decreases from $90 \%$ to $77 \%$. Fig. $2 \mathrm{~g}$ suggests that at a fixed salt concentration, the ionic conductivities of the RHE exceed those of the OHE. As salt concentration increases from 0 to $1 \mathrm{~mol} \mathrm{~L}^{-1}$, the ionic conductivity increases from 24.7 to $36.6 \mathrm{mS} \mathrm{cm}^{-1}$ for the OHE and from 24.9 to $40.8 \mathrm{mS} \mathrm{cm}^{-1}$ for the RHE. To explore the origins of enhanced conductivity and mechanical strength with increasing $\mathrm{Na}_{2} \mathrm{SO}_{4}$ concentration, low-field nuclear magnetic resonance (NMR) tests were performed for both the OHE and RHE. Samples of each were prepared to contain $1 \mathrm{~mol} \mathrm{~L}^{-1} \mathrm{Na}_{2} \mathrm{SO}_{4}$ and no added $\mathrm{Na}_{2} \mathrm{SO}_{4}$, respectively, as shown in Fig. 2h. At a fixed $\mathrm{Na}_{2} \mathrm{SO}_{4}$ concentration, the relaxation time of free water $\left(T_{2}>\right.$ $10 \mathrm{~ms}$ ) is longer in the RHE than that in the OHE, revealing that the RHE possesses lower crosslink density, accounting for its weaker mechanical strength and enhanced ionic mobility [29]. Furthermore, higher salt concentration prompts shorter relaxation time of water for both the RHE and OHE, indicating that the higher salt concentration enables tight binding of the hydrogel electrolyte with water molecules, thereby improving stability and mechanical strength [6,30]. To investigate the regeneration efficiency of cyclic utilization, we repeatedly recycled the gels, performing 5 cycles of the RHE dehydration, aerogel grinding, and the RHE formation. Fig. $2 \mathrm{i}$ shows that throughout such processing, the stiffness attenuation rate of the hydrogel electrolyte was generally maintained within $10 \%$, and the ionic conductivity slightly decreases from 40.8 to $32.8 \mathrm{mS} \mathrm{cm}{ }^{-1}$. These findings reveal that the CGs can restore considerable electrochemical and mechanical stabilities, even after 5 rounds of recycling, which might be attributed to repairable dynamic physical bonds [31].

To gain insights into the physical crosslinking within the hydrogel electrolytes, Fourier transform infrared (FT-IR) measurements for the CGs, OHE and RHE were conducted under identical experimental conditions. As shown in Fig. S7, the absorption peak at $964 \mathrm{~cm}^{-1}$ is assigned to the stretching vibration of $-\mathrm{N}\left(\mathrm{CH}_{3}\right)_{3}{ }^{+}$functional groups and the peak at $1540 \mathrm{~cm}^{-1}$ is characteristic of $\mathrm{C}=\mathrm{O}$ asymmetric stretching of the carboxylate anion. These features clearly confirm the coexistence of positive $-\mathrm{N}\left(\mathrm{CH}_{3}\right)_{3}{ }^{+}$and negative $-\mathrm{COO}^{-}$functional groups in all the samples [32,33]. Compared with the p(MAA-MAPTAC), the CGs feature an absorbance peak of $\mathrm{C}=\mathrm{O}$ stretching vibration at 
$1708 \mathrm{~cm}^{-1}$ which nearly vanished or shifted to a lower wavenumber $\left(1627 \mathrm{~cm}^{-1}\right)$ during the RHE formation, revealing the strengthening of hydrogen bonds originating from the undissociated $-\mathrm{COOH}$ groups and electrostatic interactions between ionized $-\mathrm{COO}^{-}$and protonated $-\mathrm{N}\left(\mathrm{CH}_{3}\right)_{3}{ }^{+}$groups are strengthened [34]. Additional support for these conclusions is provided by enhancement of the peak at around $3450 \mathrm{~cm}^{-1}$, which is attributed to the combined $-\mathrm{NH}$ and $-\mathrm{OH}$ stretching vibrations.

Fig. 3a gives a proposed mechanism for the RHE regeneration from the CGs. Initially, polymer chains containing $-\mathrm{COOH}$, negatively charged $-\mathrm{COO}^{-}$, and positively charged $-\mathrm{N}\left(\mathrm{CH}_{3}\right)_{3}{ }^{+}$ groups exhibit disordered distribution inside the CGs, as shown in Fig. 3a-i. Subsequent addition of water or salt solution into the CGs initiates breaking of the hydrogen bonds. With gradual diffusion of water or salt solution into the particulate gaps, salt ions bound to water molecules are absorbed onto the polymer chains. Meanwhile, the CGs begin to hydrate and swell, forming a hydration layer, wherein positive and negative charges attract each other among the CGs and give rise to non-covalent interactions (Fig. 3a-ii). Accordingly, as the viscosity of the electrolyte precursor increases, the CGs undergo microstructural changes and complete swelling in a process strongly resembling starch gelatinization. At this stage, maintaining the CGs at $60^{\circ} \mathrm{C}$ for additional $10 \mathrm{~min}$ prompts the CGs to not dissolve, but rapidly transform into the RHE (Fig. 3a-iii). Essentially, heating initiates hydrogen bond formation between the swelled CGs to thereby build a dense and integrated crosslinked network comprising the RHE. Notably, we find that the balance between hydrophilic polyion interactions and hydrogen bonds plays a key role for the RHE formation and maintaining mechanical properties during the reconstruction. Polyion interactions are a necessary precondition for hydrogel electrolyte regeneration because they induce polymer chain entanglement and subsequent rearrangement of the hydrogen bonds. We confirmed this by synthesizing a $\mathrm{p}$ (MAA-AAm) (AAm = acrylamide) hydrogel under the same experimental conditions to yield a hydrogel with no polyion interactions. The resulting $\mathrm{p}(\mathrm{MAA}-\mathrm{AAm})$ hydrogel consisted of many small pieces which could not undergo healing after breakage (Fig. S8). Fig. 3b presents micrographs of the hydrogel electrolyte in various stages of regeneration. The dark region is the CGs, while the blank region is the gap into which the salt solution is infused. Initially, the boundary between two piles of granules is evident and spacious. Two minutes after adding the salt solution, the CGs swell and merge together, leaving only a faintly discernible boundary. Extending the time after salt solution addition to $5 \mathrm{~min}$ leaves no detectable gap or defect in the RHE, suggesting the transformation of the CGs to the RHE has completed to successfully form an integrated gel electrolyte.

Considering its exceptional convenience, the process for regenerating the RHE from the CGs may enable the fabrication of smart devices such as supercapacitors from the RHE. Fig. 4a illustrates the preparation procedure for an all-gel flexible supercapacitor (Re-SC). The RHE with $1 \mathrm{~mol} \mathrm{~L}^{-1} \mathrm{Na}_{2} \mathrm{SO}_{4}$ acts as the gel electrolyte. The gel electrode was prepared by sequentially adding $0.6 \mathrm{~g}$ AC powders, $0.16 \mathrm{~g}$ conductive carbon black and $0.2 \mathrm{~g}$ CGs into $1.8 \mathrm{~g} \mathrm{Na}_{2} \mathrm{SO}_{4}$ solution $\left(0.25 \mathrm{~mol} \mathrm{~L}^{-1}\right)$ with uniform mixing. The CGs can fulfil the role typically performed by a traditional binder component. The prepared gel electrode exhibits satisfactory electronic conductivity, although excessive use of the CGs may inhibit the continuous conductivity of AC (Fig. S9). After heating at $60^{\circ} \mathrm{C}$ for $10 \mathrm{~min}$, two gel-based $\mathrm{AC}$ electrodes were tightly adhered onto the upper and lower surfaces of the RHE due to their excellent compatibility. The scanning electron microscopy (SEM) image in Fig. 4b clearly demonstrates that the Re-SC features even assembly, without any detectable defects or gaps at the interface between electrode and electrolyte. This may be attributed to the tendency of hydrogen bonds and polyion interactions in the RHE to promote adhesion of the electrode and electrolyte into an integrated polymer network. Fig. 4c exhibits that the Re-SC is flexible and can be hand-pinched into specific shapes.

The electrochemical properties of the Re-SC were systematically investigated via cyclic voltammetry $(\mathrm{CV})$, galvanostatic charge-discharge (GCD), and electrochemical impedance spectroscopy (EIS) measurements. The nearly rectangular CV curves in Fig. 4d signify typical capacitive behaviours of the Re-SC over scan rates ranging from 5 to $100 \mathrm{mV} \mathrm{s}^{-1}$. Meanwhile, the nearly

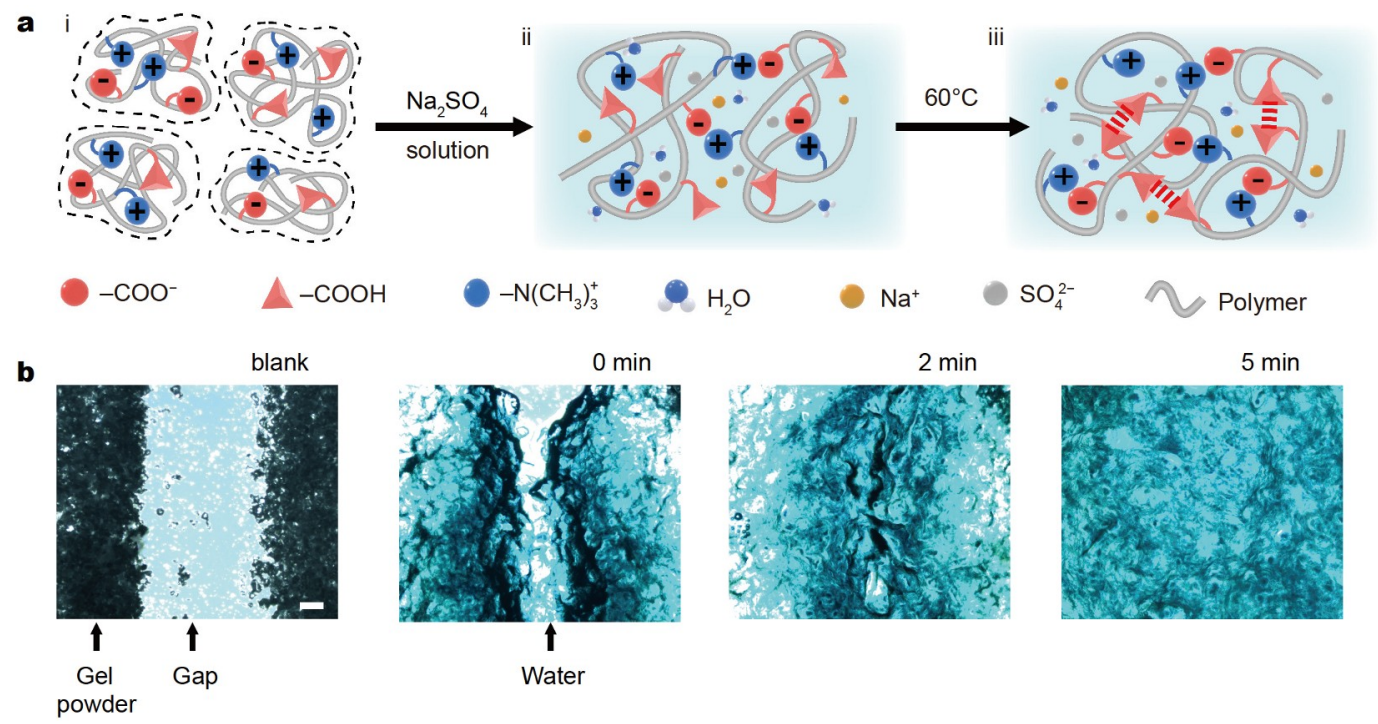

Figure 3 (a) Proposed regeneration mechanism of the RHE. (b) Regeneration process from the CGs as observed by an optical microscope (scale bar: $200 \mu \mathrm{m})$. 
a $\quad$ Add $\mathrm{Na}_{2} \mathrm{SO}_{4}$ solution
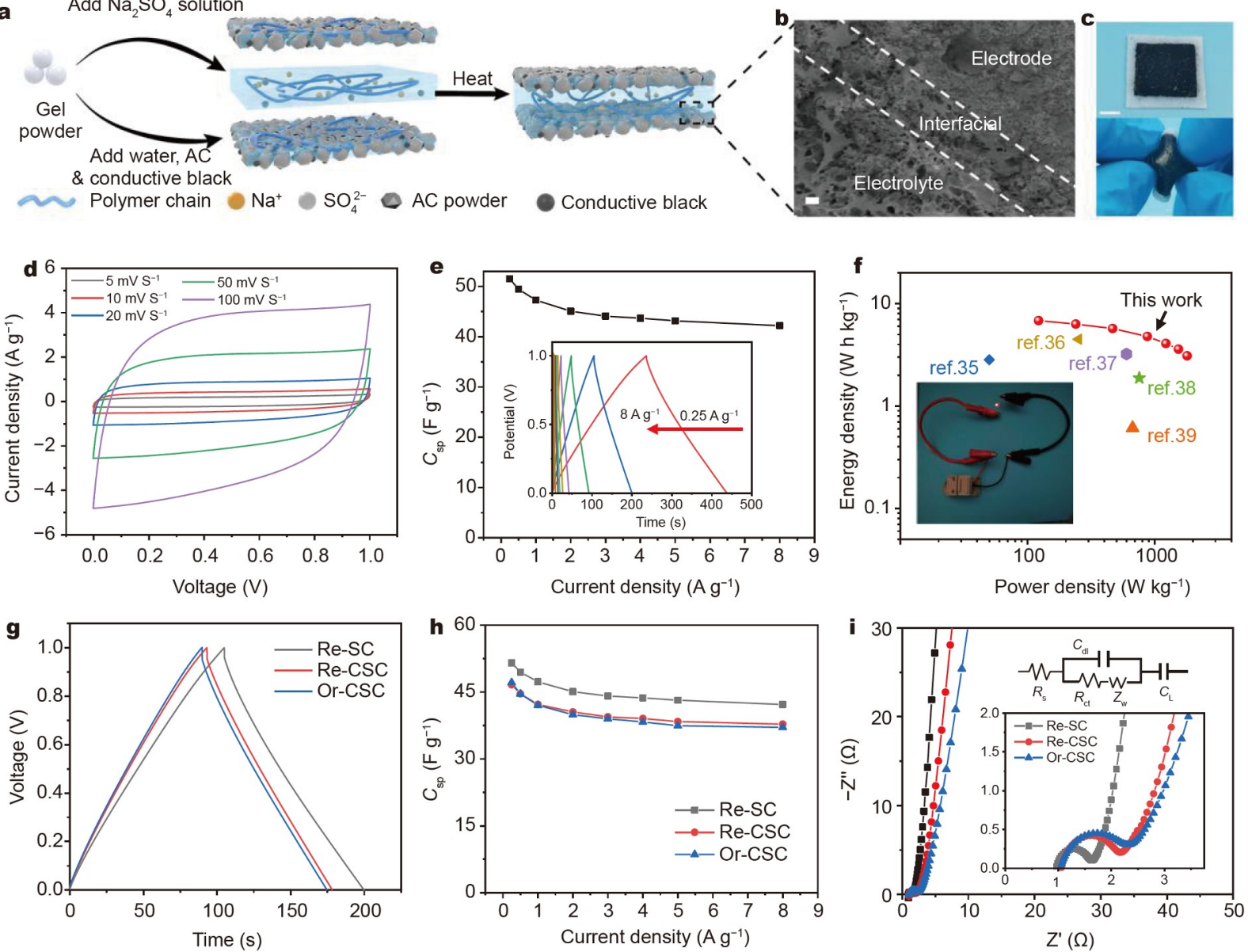

Figure 4 Preparation and electrochemical performance of the Re-SC. (a) Schematic of preparation procedure. (b) SEM image of the interface between the RHE and electrode in the Re-SC capacitor (scale bar: $500 \mu \mathrm{m}$ ). (c) The Re-SC supercapacitor can be shaped by hand. (d) CV curves of the Re-SC at various scan rates. (e) $C_{s p}$ of the Re-SC at various current densities. Inset: GCD curves with different current densities. (f) Ragone plot of the Re-SC. Inset: red LED lamp powered by the Re-SC. (g-i) GCD curves, $C_{\mathrm{sp}}$ and Nyquist plots of the Re-SC, Re-CSC and Or-CSC.

linear and symmetric character of the GCD profiles at different current densities, depicted in Fig. 4e, reveals that the Re-SC has a nearly ideal capacitive character in the current densities ranging from 0.25 to $8.0 \mathrm{~A} \mathrm{~g}^{-1}$. The highest specific capacitance $\left(C_{\mathrm{sp}}\right)$ of the device is $51.5 \mathrm{~F} \mathrm{~g}^{-1}$, which is obtained at a current density of $0.25 \mathrm{~A} \mathrm{~g}^{-1}$. However, even at a current density of $8.0 \mathrm{~A} \mathrm{~g} \mathrm{~g}^{-1}$, the Re-SC delivers a $C_{\text {sp }}$ of $42.2 \mathrm{~F} \mathrm{~g}^{-1}$, which is competitive with similar flexible devices. Furthermore, the Re-SC features satisfactory capacitive properties, as suggested by the data in Fig. $4 \mathrm{f}$ and Table S3. For example, in the range of current densities from 0.25 to $8.0 \mathrm{~A} \mathrm{~g}^{-1}$, the calculated energy and power density values are acceptable compared with those from the references under similar experimental conditions [35-39]. To demonstrate the effectiveness of the Re-SC, supercapacitors composed of commercial AC electrodes and either the RHE (Re-CSC) or the OHE (Or-CSC) were assembled respectively, while polytetrafluoroethylene was employed as the binder for the commercial AC electrodes. Fig. $4 \mathrm{~g}$ shows that electrochemical properties of the Re-SC outperform those of the other two supercapacitors. The discharge time for the Re-SC is $200 \mathrm{~s}$, which exceeds those of the Re-CSC (178 s) and the Or-CSC (175 s). Fig. 4h shows that as the current density increases from 0.5 to $8.0 \mathrm{~A} \mathrm{~g}^{-1}$, the value of $C_{\mathrm{sp}}$ decreases only by $7.24 \mathrm{~F} \mathrm{~g}^{-1}$, a quantity equal to about $15 \%$ of the highest achieved capacitance $\left(49.4 \mathrm{~F} \mathrm{~g}^{-1}\right.$ at $\left.0.5 \mathrm{~A} \mathrm{~g}^{-1}\right)$, demonstrating excellent rate capability. From the respective Nyquist curves in Fig. 4i, the charge transfer resistance $\left(R_{\mathrm{ct}}\right)$ is calculated to be $0.6 \Omega$ for the Re-SC, $1.1 \Omega$ for the Re-CSC, and $1.3 \Omega$ for the Or-CSC, suggesting that among the three supercapacitors based on hydrogel electrolytes, the RHE with the AC/ CGs composite electrodes shows the best interfacial compatibility. Fig. S10 indicates that the device can withstand more than 1000 electrochemical testing cycles without suffering significant loss of electrometrical performance. We acknowledge that the long-term stability of the Re-SC is still inferior to those reported in the literatures, leaving opportunities for further study and improvements.

By leveraging the flexibility and reprocessability of the Re-SC, we studied the effect of bending on the capacitance. As shown in Fig. $5 \mathrm{a}$ and $\mathrm{b}$, the CV and GCD curves are consistent across different bending states $\left(0^{\circ}, 90^{\circ}, 180^{\circ}\right.$ and twist) (Fig. $\left.5 \mathrm{c}\right)$. The Nyquist curves (Fig. S11) indicate that internal impedance does not change significantly during bending, and changes only slightly after twisting, suggesting that the bond between the hydrogel electrolyte and electrodes is stable [40]. Fig. 5d and Fig. S12 indicate that even after 100 bending cycles, the Re-SC can achieve $98.7 \%$ of its original $C_{\mathrm{sp}}$, meanwhile maintaining its $\mathrm{CV}$ and GCD curve shapes and showing excellent mechanical stabilities. Another important property of supercapacitors is 

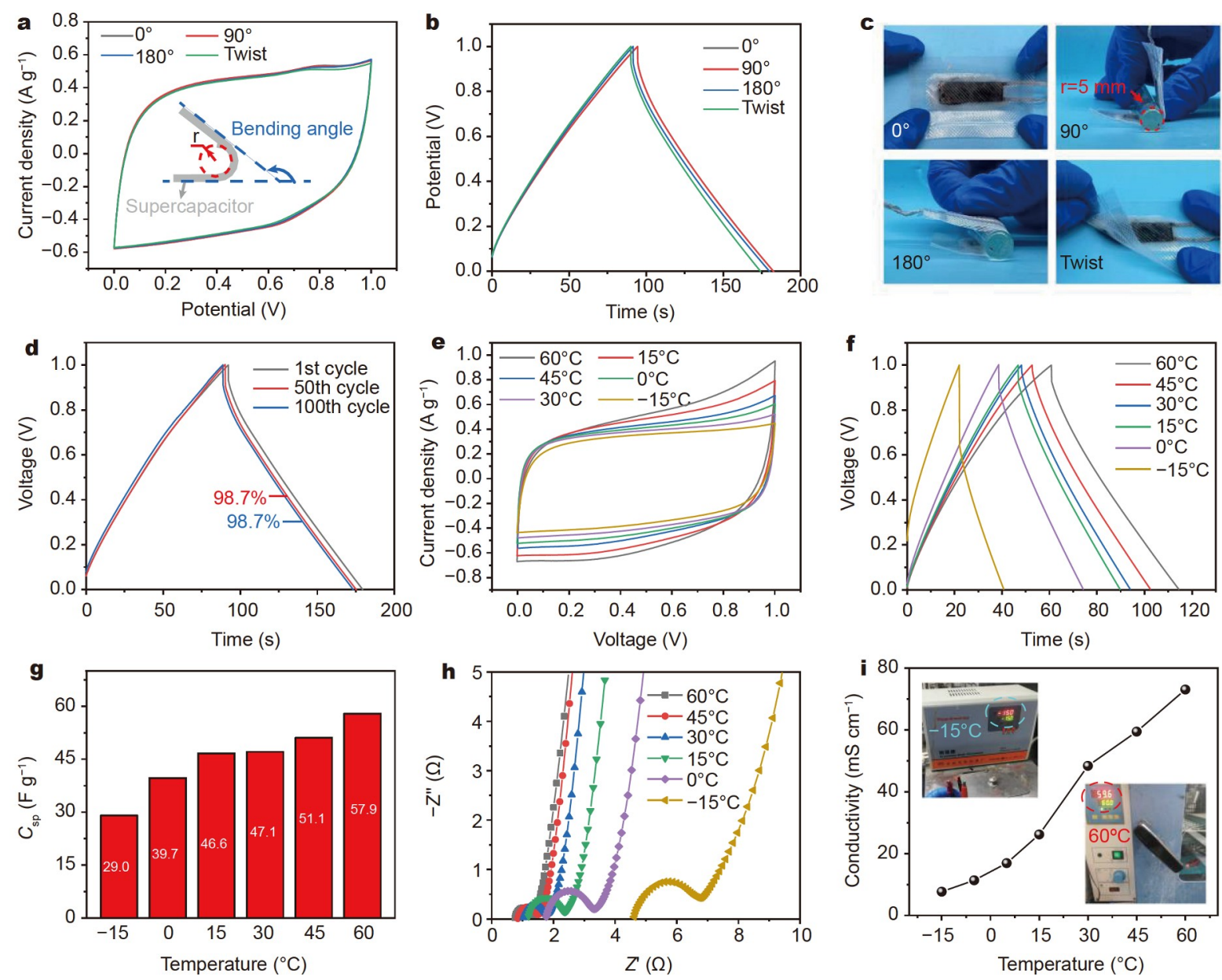

Figure 5 Flexibility and temperature tolerance of the Re-SC. (a, b) CV curves, GCD curves of the Re-SC at different bending states. (c) Photos of the device at different bending states. (d) GCD curves of the Re-SC after different bending cycles. (e) Temperature dependence of the CV performance at various temperatures. (f-h) GCD curves, $C_{\mathrm{sp}}$ and Nyquist plots of the Re-SC in the range of -15 to $60^{\circ} \mathrm{C}$. (i) Ionic conductivity of the RHE under different temperatures. Inset: red LED lamp powered by the Re-SC at -15 and $60^{\circ} \mathrm{C}$.

their stability to temperature, which affects their use in a wide range of applications, and therefore drives strong demand for hydrogel electrolytes suitable for extreme conditions [41]. The satisfactory water retention capacity of the RHE at high temperatures after being packaged facilitates normal operation of the device (Fig. S13). Fig. 5e shows that all CV curves are rectangular in the -15 to $60^{\circ} \mathrm{C}$ temperature range. Fig. $5 \mathrm{f}$ demonstrates that all GCD profiles are nearly linear and symmetric, revealing that the $\mathrm{Re}-\mathrm{SC}$ can operate normally in this temperature range. Fig. $5 \mathrm{~g}$ illustrates that the $C_{\mathrm{sp}}$ gradually increases with increasing temperatures, achieving maximum and minimum $C_{\mathrm{sp}}$ values of $57.9 \mathrm{~F} \mathrm{~g}^{-1}$ at $60^{\circ} \mathrm{C}$ and $29.0 \mathrm{~F} \mathrm{~g}^{-1}$ at $-15^{\circ} \mathrm{C}$, respectively. EIS plots in Fig. $5 \mathrm{~h}$ confirm that high temperature is beneficial for the reduction of bulk resistance $\left(R_{\mathrm{b}}\right)$ and $R_{\mathrm{ct}}$, which may be attributable to accelerated ion migration and diffusion within the polymer matrix by high temperatures. Further, the Re-SC exhibits acceptable stability at high and low temperatures. After $10 \mathrm{~h}$ at either -15 or $50^{\circ} \mathrm{C}$, the capacitance remains at $99.8 \%$ and $86.7 \%$, respectively (Fig. S14). As a practical demonstration, Fig. 5i vividly depicts that the light-emitting diode (LED) bulb can be lighted by the Re-SC at selected temperatures, suggesting promising application of the Re-SC in extreme environments. The leakage current and self-discharge of the Re-SC shown in
Fig. S15 demonstrate satisfactory electrochemical properties for practical application. These findings offer valuable insights into the quick and effective fabrication of novel energy storage devices.

\section{CONCLUSIONS}

In summary, we report a facile strategy for the regeneration of hydrogel electrolytes from their CGs via direct addition of water or salt solution. This transformation was exceptionally convenient, rapid, and efficient. The CGs composed of MAPTAC and MAA were lightweight, stable under long-term storage, and capable of being recycled multiple times. The synergistic effect between polyion interactions and hydrogen bonds played a crucial role for the regeneration of the hydrogel electrolytes and maintenance of their mechanical strength. After 5 cycles of dehydration and regeneration, the regeneration efficiency of the RHE was sustained at more than $60 \%$, while the ionic conductivity was maintained at $80 \%$. Considering the everexpanding breadth of energy storage applications of hydrogel electrolytes, we fabricated an all-gel-based flexible supercapacitor comprised of an RHE and AC/CGs composite electrodes. The all-gel supercapacitor was demonstrated to be suitable across a wide temperature range spanning from -15 to 
$60^{\circ} \mathrm{C}$, exhibiting acceptable capacitance of the device (29.0$57.9 \mathrm{~F} \mathrm{~g}^{-1}$ ) at a current density of $0.5 \mathrm{~A} \mathrm{~g}^{-1}$. The RHE displayed high compatibility with the composite gel electrode, facilitating the device to hold $98.7 \%$ of its original $C_{\mathrm{sp}}$ even after 100 bending cycle operations, thereby revealing satisfactory electrochemical and mechanical stability. This work is expected to provide a new platform for the development of recyclable and renewable materials with various applications in fields including intelligent devices, flexible/wearable electronics and soft robotics.

\section{Received 18 March 2021; accepted 21 May 2021; published online 16 July 2021}

1 Huang J, Peng S, Gu J, et al. Self-powered integrated system of a strain sensor and flexible all-solid-state supercapacitor by using a high performance ionic organohydrogel. Mater Horiz, 2020, 7: 2085-2096

2 Huang Y, Tang Z, Liu Z, et al. Toward enhancing wearability and fashion of wearable supercapacitor with modified polyurethane artificial leather electrolyte. Nano-Micro Lett, 2018, 10: 38

3 Guo T, Zhou D, Liu W, et al. Recent advances in all-in-one flexible supercapacitors. Sci China Mater, 2021, 64: 27-45

4 Guo Y, Bae J, Zhao F, et al. Functional hydrogels for next-generation batteries and supercapacitors. Trends Chem, 2019, 1: 335-348

5 Tai Z, Wei J, Zhou J, et al. Water-mediated crystallohydrate-polymer composite as a phase-change electrolyte. Nat Commun, 2020, 11: 1843

6 Wei J, Wei G, Shang Y, et al. Dissolution-crystallization transition within a polymer hydrogel for a processable ultratough electrolyte. Adv Mater, 2019, 1900248

7 Han X, Xiao G, Wang Y, et al. Design and fabrication of conductive polymer hydrogels and their applications in flexible supercapacitors. J Mater Chem A, 2020, 8: 23059-23095

8 Wang Z, Pan Q. An omni-healable supercapacitor integrated in dynamically cross-linked polymer networks. Adv Funct Mater, 2017, 27: 1700690

9 Li H, Lv T, Sun H, et al. Ultrastretchable and superior healable supercapacitors based on a double cross-linked hydrogel electrolyte. Nat Commun, 2019, 10: 536

10 Jia R, Li L, Ai Y, et al. Self-healable wire-shaped supercapacitors with two twisted $\mathrm{NiCo}_{2} \mathrm{O}_{4}$ coated polyvinyl alcohol hydrogel fibers. Sci China Mater, 2018, 61: 254-262

11 Zang L, Liu Q, Qiu J, et al. Design and fabrication of an all-solid-state polymer supercapacitor with highly mechanical flexibility based on polypyrrole hydrogel. ACS Appl Mater Interfaces, 2017, 9: 33941-33947

12 Wang K, Zhang X, Li C, et al. Chemically crosslinked hydrogel film leads to integrated flexible supercapacitors with superior performance. Adv Mater, 2015, 27: 7451-7457

13 Li H, Han C, Huang Y, et al. An extremely safe and wearable solid-state zinc ion battery based on a hierarchical structured polymer electrolyte. Energy Environ Sci, 2018, 11: 941-951

14 Zhou J, Wu D, Wu C, et al. Diffusion-determined assembly of allclimate supercapacitors via bioinspired aligned gels. J Mater Chem A, 2019, 7: 19753-19760

15 Peng X, Liu H, Yin Q, et al. A zwitterionic gel electrolyte for efficient solid-state supercapacitors. Nat Commun, 2016, 7: 11782

16 Wu S, Shao Z, Xie H, et al. Salt-mediated triple shape-memory ionic conductive polyampholyte hydrogel for wearable flexible electronics. J Mater Chem A, 2021, 9: 1048-1061

17 Mo F, Chen Z, Liang G, et al. Zwitterionic sulfobetaine hydrogel electrolyte building separated positive/negative ion migration channels for aqueous $\mathrm{Zn}-\mathrm{MnO}_{2}$ batteries with superior rate capabilities. $\mathrm{Adv}$ Energy Mater, 2020, 10: 2000035

18 Shang Y, Wu C, Hang C, et al. Hofmeister-effect-guided ionohydrogel design as printable bioelectronic devices. Adv Mater, 2020, 32: 2000189

19 Gao Y, Shi L, Lu S, et al. Highly stretchable organogel ionic conductors with extreme-temperature tolerance. Chem Mater, 2019, 31: 3257-3264

20 Yadav N, Hashmi SA. Energy enhancement of quasi-solid-state su- percapacitors based on a non-aqueous gel polymer electrolyte via a synergistic effect of dual redox additives diphenylamine and potassium iodide. J Mater Chem A, 2020, 8: 18266-18279

21 Luo F, Sun TL, Nakajima T, et al. Free reprocessability of tough and self-healing hydrogels based on polyion complex. ACS Macro Lett, 2015, 4: 961-964

22 Dai L, Zhang L, Wang B, et al. Multifunctional self-assembling hydrogel from guar gum. Chem Eng J, 2017, 330: 1044-1051

23 Yang Y, Guan E, Zhang T, et al. Influence of water addition methods on water mobility characterization and rheological properties of wheat flour dough. J Cereal Sci, 2019, 89: 102791

24 Liu R, Xing Y, Zhang Y, et al. Effect of mixing time on the structural characteristics of noodle dough under vacuum. Food Chem, 2015, 188: 328-336

25 Farahnaky A, Hill SE. The effect of salt, water and temperature on wheat dough rheology. J Texture Studies, 2007, 38: 499-510

26 Luo F, Sun TL, Nakajima T, et al. Oppositely charged polyelectrolytes form tough, self-healing, and rebuildable hydrogels. Adv Mater, 2015, 27: 2722-2727

27 Qu G, Li Y, Yu Y, et al. Spontaneously regenerative tough hydrogels. Angew Chem Int Ed, 2019, 58: 10951-10955

28 Mazzotta MG, Putnam AA, North MA, et al. Weak bonds in a biomimetic adhesive enhance toughness and performance. J Am Chem Soc, 2020, 142: 4762-4768

29 Assifaoui A, Champion D, Chiotelli E, et al. Characterization of water mobility in biscuit dough using a low-field ${ }^{1} \mathrm{H}$ NMR technique. Carbohydrate Polyms, 2006, 64: 197-204

$30 \mathrm{Wu}$ J, Lin W, Wang Z, et al. Investigation of the hydration of nonfouling material poly(sulfobetaine methacrylate) by low-field nuclear magnetic resonance. Langmuir, 2012, 28: 7436-7441

31 Wang MX, Chen YM, Gao Y, et al. Rapid self-recoverable hydrogels with high toughness and excellent conductivity. ACS Appl Mater Interfaces, 2018, 10: 26610-26617

32 Du Q, Tang Q, Yang K, et al. One-step preparation of tough and selfhealing polyion complex hydrogels with tunable swelling behaviors. Macromol Rapid Commun, 2019, 40: 1800691

33 Wei J, Zhou J, Su S, et al. Water-deactivated polyelectrolyte hydrogel electrolytes for flexible high-voltage supercapacitors. ChemSusChem, 2018, 11: 3410-3415

34 Lei $\mathrm{Z}, \mathrm{Wu}$ P. A supramolecular biomimetic skin combining a wide spectrum of mechanical properties and multiple sensory capabilities. Nat Commun, 2018, 9: 1134

35 Liu Y, Weng B, Razal JM, et al. High-performance flexible all-solidstate supercapacitor from large free-standing graphene-PEDOT/PSS films. Sci Rep, 2015, 5: 17045

36 Park JH, Rana HH, Lee JY, et al. Renewable flexible supercapacitors based on all-lignin-based hydrogel electrolytes and nanofiber electrodes. J Mater Chem A, 2019, 7: 16962-16968

37 Liu Y, Zhou J, Chen L, et al. Highly flexible freestanding porous carbon nanofibers for electrodes materials of high-performance all-carbon supercapacitors. ACS Appl Mater Interfaces, 2015, 7: 23515-23520

38 Chen X, Qiu L, Ren J, et al. Novel electric double-layer capacitor with a coaxial fiber structure. Adv Mater, 2013, 25: 6436-6441

39 Xu Y, Lin Z, Huang X, et al. Flexible solid-state supercapacitors based on three-dimensional graphene hydrogel films. ACS Nano, 2013, 7: 4042-4049

$40 \mathrm{Li} \mathrm{H}$, Tang Z, Liu Z, et al. Evaluating flexibility and wearability of flexible energy storage devices. Joule, 2019, 3: 613-619

41 Liu L, Dou Q, Sun Y, et al. A moisture absorbing gel electrolyte enables aqueous and flexible supercapacitors operating at high temperatures. J Mater Chem A, 2019, 7: 20398-20404

Acknowledgements This work was financially supported by the National Key R\&D Program of China (2018YFC1803100 and 2016YFA0100800), and the National Natural Science Foundation of China (51873156).

Author contributions $\mathrm{He} \mathrm{X}, \mathrm{Wu} \mathrm{D}$ and Wang Q designed the studies and prepared the manuscript. He X carried out most of the experiments in- 
cluding the preparation and characterization of the material, manufacturing and electrochemical measurement of the energy storage devices. Wu D and He X wrote the original draft. Wang Q, Wang X and Li W revised the draft elaborately. Shang Y made supporting contribution in the electrochemical measurement. Shen $\mathrm{H}$ and Xi S made supporting contribution in the material characterization. All authors discussed the results and commented on the manuscript.

Conflict of interest The authors declare that they have no conflict of interest.

Supplementary information Experimental details and supporting data are available in the online version of the paper.

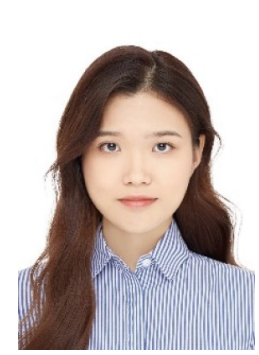

Xian He is a master student at the School of Chemical Science and Engineering, Tongji University. She received her BE degree from Donghua University. Her research interest focuses on hydrogel electrolytes and flexible supercapacitors.

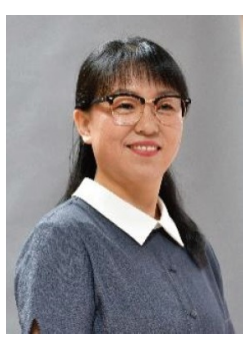

Dongbei $\mathbf{W u}$ is an associate professor at the School of Chemical Science and Engineering, Tongji University. She received her $\mathrm{PhD}$ degree from Changchun Institute of Applied Chemistry, Chinese Academy of Sciences in 2005. Her research interest focuses on hydrogel electrolytes and flexible supercapacitors.

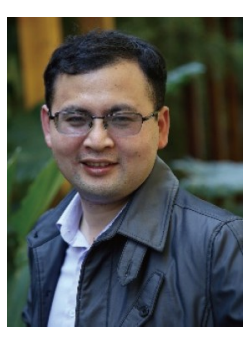

Qigang Wang is currently a professor at the School of Chemical Science and Engineering, Tongji University. He received his $\mathrm{PhD}$ degree from Shanghai Institute of Ceramics, Chinese Academy of Sciences in 2005. He was the postdoctor of Hong Kong University of Science and Technology, The University of Tokyo and Riken in 2005-2011. His research interest focuses on enzymatic polymerized methodology and hydrogel electrolyte design for flexible electronics/bioelectronics.

\section{可再生水凝胶电解质用于全凝胶超级电容器}

何弦, 乌东北 ${ }^{*}$, 尚英辉, 沈红豆, 奚松延, 王霞, 李汶军, 王启刚 ${ }^{*}$

摘要 再生电解质对环境保护和可持续发展具有重要意义. 受面粉加 水成面团的启发，本工作提出一种简单、快速制备凝胶电解质的策略， 该再生水凝胶电解质可以通过向其脱水的共聚物颗粒中加入水或盐溶 液, 经过混合、加热即可获得. 脱水的共聚物颗粒质量小、性质稳定、 可长期储存和多次循环使用. 经过 5 次脱水-再生的循环后, 凝胶电解质 的力学强度仍保持 $60 \%$ 以上. 该再生凝胶电解质具有高离子电导, 可再 加工, 并可用于 $3 \mathrm{D}$ 打印. 同时以凝胶共聚物颗粒为粘合剂的活性炭电 极、再生凝胶为电解质组装的全凝胶超级电容器具有极佳的界面相容 性, 在 100 次弯曲试验后仍能保持 $98.7 \%$ 的比电容, 并可在 -15 至 $60^{\circ} \mathrm{C}$ 的 宽温度范围内工作．这项工作为可再生材料的制备及其应用提供了新 的途径. 